\title{
Secure and Intelligent QoS aware Routing Protocol for VANET Environment
}

\author{
S. Gayathri' ${ }^{1}$ J. Granty Regina Elwin ${ }^{2 *}$, Reshma B. Nair'², K. Ranjeethapriya ${ }^{2}$ and R. Ramesh Kumar ${ }^{2}$ \\ 'Department of Computer Science and Engineering, Amrita School of Engineering, \\ Amrita University, Coimbatore - 641112, Tamilnadu, India; gayathri24coolgal@gmail.com \\ 2Department of Information Technology, Sri Krishna College of Engineering and Technology, \\ Coimbatore -641008,Tamilnadu, India; grantyregina@gmail.com, reshmab@skcet.ac.in, \\ ranjeethapriyak@skcet.ac.in, ramesh4477@gmail.com
}

\begin{abstract}
Objectives: To propose a suitable protocol that is secured and efficient for data transmission in Vehicular Adhoc Networks (VANET) environment. Methods/Statistical Analysis: The main challenge in VANET is to protect valuable information from the users. However there are many malicious vehicles that affect the system. To overcome this problem, the Secure and Intelligent routing (SIR) protocol is used that will transmit the data in shortest path through the authenticated vehicles. The proposed method makes use of message transmission, link connectivity and vehicle positioning to forward the data to the destination. Sending the data through the QoS based optimized path will enhance the performance of the system. Hence the proposed Enhanced Secure and intelligent routing (ESIR) protocol is QoS enabled and optimized. The QoS metrics taken into concern are delay, network load and distance with minimum number of hops. Particle Swarm Optimization (PSO) algorithm used for optimization in the proposed work takes one main parameter into concern; it is distance with minimum number of hops. Findings: The proposed technique is compared with the existing SIR protocol in terms of throughput, delay, packet delivery ratio, residual energy levels and packet drop. The results obtained show that the proposed technique serves as an efficient routing protocol for VANET environment providing a reliable and robust service.
\end{abstract}

Keywords: Particle Swarm Optimization, QoS Parameters, Shortest Path, VANET

\section{Introduction}

VANET (Vehicular Adhoc Networks) is a network with dynamic topology where nodes move at fast speed. It has more processing power, storage and energy than handhelds. In general, VANET uses location based information to detect any accidents that occur in the network. In VANET, the communication in the network is of two types. They are Vehicle-to-Vehicle communication $(\mathrm{v} 2 \mathrm{v})$ and Vehicle-to-Infrastructure communication (v2I). In our environment we use both $\mathrm{v} 2 \mathrm{v}$ as well as v2I communications.

According to Figure 1, the vehicles communicate with each other and they communicate with the Infrastructure too. Hence they use both v2v and v2I communications. The main challenge is to reduce the delay constraint during communication in the network, by deploying intelligence.
There are many goals in VANET. They are

- VANET can be used to improve traffic safety and provide comfort for the drivers.

- The main goal of VANET is to minimize accidents and traffic intensity.

- VANET must provide up-to-date traffic information.

- VANET can provide weather information as well as vacancies in the parking lots.

All these goals can be achieved by sending data to all the vehicles in the network. Hence we must ensure that the data sent is secured.

In this study we will propose a protocol that is secure and is efficient for data transmission in VANET environment. To begin with, Section II explains some existing works about the routing protocols in VANET environment. Section III is the detailed work plan of the existing work. Section IV is the detailed plan of the 


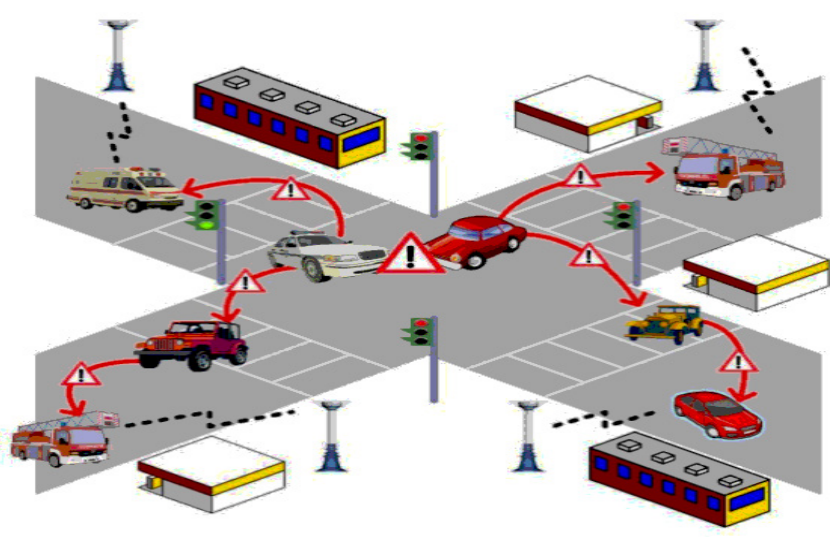

Figure 1. Vehicular networks communication.

proposed work. Section V is the simulation. Section VI presents the result and analysis of the proposed work. Finally, Section VII gives the conclusion.

\section{Literature Survey}

Many efficient routing protocols (Broadcast, Geocast, Clustering, Position-based and beaconing) are proposed for VANET 1,2 .

$\mathrm{In}^{1,3}$ planned a greedy traffic aware routing (GyTAR) protocol to send the information expeditiously. This protocol uses the cell information packet to seek out score of its neighboring junction. However, this suffers from congestion as a result of the cell information packets area unit changed at high rate. Moreover, it's an insecure routing protocol that's full of the attackers within the network.

Anchor-based street and traffic aware routing (A-star) $)^{1,4}$ could be a routing protocol wherever the information is forwarded in a very pre-defined path. The most disadvantage of this protocol is, it lacks current traffic info.

Securing the optimized link state routing (SOLSR) $)^{1,5}$ could be a secure OLSR protocol that's accustomed give authentication to the information packets and helps in protective the network from replay attacks. It uses symmetrical key cryptography that helps in reducing computation overhead within the network. However this protocol lacks data regarding the network gaps and conjointly will increase delay within the network.

Time efficient Stream loss-tolerant authentication $\frac{1,6}{}$ could be a broadcast authentication wherever HMAC is employed for security. This lacks data regarding network gaps and thus will increase path length also as delay within the network.
A QoS-based clustering protocol named as VANETQoS-OSLR is planned 7,8 . The goal is to create stable clusters and maintain stability and link property within the network. This routing protocol is evaluated with ACO (Ant-colony optimization) algorithmic program. The most disadvantage is it will increase delay within the network.

\section{Secure and Intelligent Routing (SIR) Protocol}

In SIR model, a Trusted Vehicle (TV) 1 is fixed at every Junction (location where multiple roads intersect). This TV will store beacon messages like vehicle id, speed, location and direction ${ }^{1}$. Hence, this TV will act as an intelligent system by communicating in the network.

Initially, the vehicles in the network exchange beacon messages at specific intervals to know their neighbouring vehicles. Vehicles in the network use Global position system service to know their own location 1 . The functionality of SIR starts by selecting a source vehicle $\mathrm{V}_{\mathrm{s}}$ and destination vehicle $\mathrm{V}_{\mathrm{D}}$. The source vehicle $\mathrm{V}_{\mathrm{s}}$ will contain data that has to be sent to the destination $V_{D}$ in short time. To forward the data, $\mathrm{V}_{\mathrm{s}}$ first finds the shortest path to destination by using Dijkstra's Algorithm² by considering the junctions ${ }^{1}$. Dijkstra's algorithm is used since it has less time complexity than any other shortest path algorithms. $\mathrm{V}_{\mathrm{s}}$ forward the encrypted data to the neighbouring vehicles only after the verification of their genuineness. The Central Authority (CA) will provide valid certificate to the authenticated vehicles. Hence, the vehicles in the neighbouring field will verify the vehicle by validating the certificate 'C'. Vehicle with valid $C$ is genuine vehicle while vehicles with invalid $C$ are malicious vehicles ${ }^{1}$. Once again for more security reasons, the data that is being forwarded should not be altered during transmission. Hence the message is secured and protected by using Diffie-Hellman Key agreement protocol ${ }^{10}$. The vehicles will forward the encrypted data to the neighbouring genuine vehicles until it reaches the junction. The vehicle closest to the junction will forward the encrypted data to the TV. (TV is assumed to be trusted with previous history). The $\mathrm{TV}$ will calculate a weight $\mathrm{W}$ for every neighbouring junction and will forward the data to the junction that has minimum weight $\mathrm{W}^{\underline{11}}$.

The calculation of weight $\mathrm{W}$ is: 
$\mathrm{W}=$ Shortest distance from current junction to the destination + total number of genuine vehicles + time of transmission between source and destination + total number of link connectivity between the vehicles.

Link connectivity $=$ It is the link availability time of the vehicle in the network (the total time the vehicle is available in the network).

Hence, by calculating the weight $\mathrm{W}$ for each path, the TV will forward the data to the path that has minimum $\mathrm{W}^{1}$. This process will continue until the encrypted data reaches the destination vehicle $V_{D}$. The destination vehicle will decrypt the data and will take proper decision.

\section{Methodology - Sir with QoS and Pso}

From the existing SIR protocol (including Intelligent system (TV), beacon message for neighbour discovery, 'C' certificate), the proposed Enhanced Secure and Intelligent routing (ESIR) protocol adds up QoS metrics and Optimization concept.

In the existing SIR protocol for encryption and decryption of messages, Diffie-Hellman key exchange protocol was considered. The main drawback of DiffieHellman Key exchange protocol is the private key is smaller which can be easily decoded. Hence the proposed (ESIR) protocol uses RC4 (Ron's Code 4) algorithm for encryption and decryption of messages. Encryption works by running data through a Key. Both the sender and receiver should know this key for encrypting and decrypting the data.

The steps for RC4 algorithmic program is:

- Obtain the info to be encrypted and therefore the secret key.

- Generate 2 string arrays.

- Fill one array with numbers from zero to 255 .

- Fill the opposite array with the key.

- Randomize the primary array betting on the array (second array) of the key.

- Randomize the primary array at intervals itself to come up with the ultimate key stream of Knowledge.

- XOR the ultimate key stream of knowledge with the info to be encrypted to offer cipher text.

The enhanced SIR (ESIR) considers three QoS metrics.

They are delay, network load and distance with minimum number of hops. By considering these metrics, all possible paths from source to destination are obtained. From the above paths, we perform Particle Swarm Optimization (PSO) with distance between source and destination as parameter to get the best shortest path.

From all possible paths from source to destination, the ESIR protocol chooses path that has minimum delay with threshold less than $40 \%$. From the chosen paths, the ESIR protocol again chooses path that have minimum network load with threshold less than $70 \%$.

Particle Swarm Optimization (PSO) algorithm is used to identify feasible routes between source and destination. From the above selected paths, the algorithm finds the path that has minimum distance with less number of hops. Hence, the path having minimum distance with less number of hops is taken as the final path that can be used to traverse from source to destination. The basic concept of PSO is to iterate between the paths and find the best feasible path from source and destination.

The proposed work is:

- Initializing the system

- Computing all paths from source to destination

- Selecting paths that have minimum delay with threshold less than $40 \%$

- From the above selected paths, choose paths with network load less than $70 \%$

- Initialize the selected paths to a new array A[ ]

- For I = 1 to ITER

- Generating particles from A[ ]

- checking path that has minimum distance with less number of hops

- Updating the best path with less distance

- End for

By doing this we achieve a secure feasible path in the network.

\section{Simulation Results}

The simulation is implemented in NS2 (Network Simulator). Simulation Results for the various parameters employed for performance evaluation is listed in Table 1.

The simulation environment consists of 5 junctions $(12,13,14,15$, and 16) as shown in Figure 2. Source and destination points are received from the terminal. Once the source and destination is known we generate keys for individual vehicles as well as for the TV's. The keys generated are taken as session keys for communication between the vehicles in the network. 
Table 1. Simulation for Performance evaluation

\begin{tabular}{|l|c|}
\hline \multicolumn{1}{|c|}{ PARAMETERS } & $\begin{array}{c}\text { PARAMETER } \\
\text { VALUES }\end{array}$ \\
\hline Number of junctions & 5 \\
\hline Vehicle speed & $40-80 \mathrm{~km} / \mathrm{hr}$ \\
\hline Vehicle range & $250 \mathrm{~m}$ \\
\hline TV range & $250 \mathrm{~m}$ \\
\hline Number of RSU & 4 \\
\hline Number of vehicles & 30 \\
\hline Packet size & 512 bytes \\
\hline
\end{tabular}

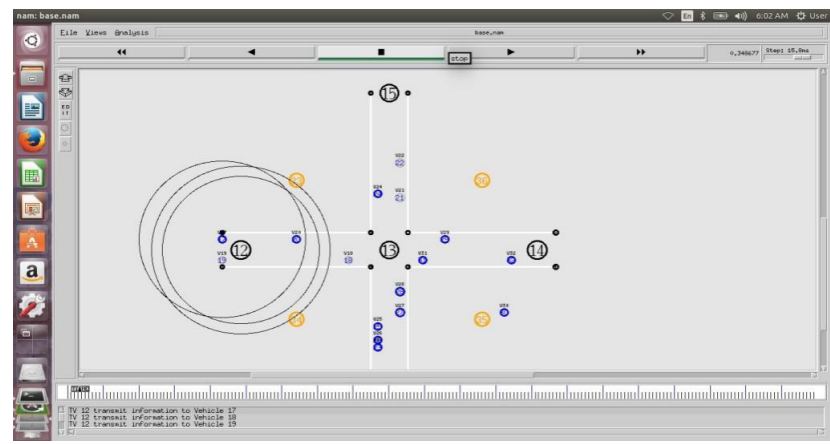

Figure 2. Simulation Environment.

Figure 3 shows the keys for individual vehicles and for TV's. The keys are generated randomly and are assigned for each vehicle and TV. With the help of these session keys, the vehicle will encrypt and decrypt data in the network. TV just transmits the data without decrypting. We use RC4 algorithm for encryption. Overhead of key generation between vehicle and TV are lesser as only one key is generated for one session from source to destination. Encryption and decryption is done end-to-end.

When the user enters the source and destination, the vehicles in the network will exchange beacon messages to know their neighbouring vehicles in the network. With this information, the TV at the junction will calculate the

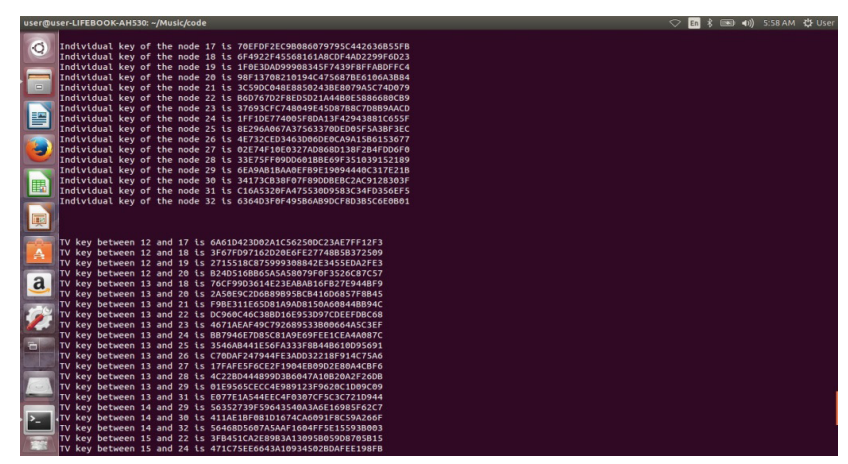

Figure 3. Keys for vehicles and TV. weight $\mathrm{W}$ for all the neighbouring TV's and will choose the path that has minimum W.

In this simulation, the user entered 17 as source vehicle with message "accident" and has to be transmitted to the destination 27. Now the nearest TV for 17 is 12 . Hence vehicle 17 will forward the encrypted data to TV 12. Now TV 12 will calculate $\mathrm{W}$ for the neighbouring TV's and TV 12 found that TV 13 is having minimum $\mathrm{W}$ than other TV's. Hence the data is forwarded to TV 13. Then TV 13 found that the destination vehicle 27 is nearby. Hence, it forwards the encrypted data to the destination and decryption is carried out by vehicle 27 . The execution of this process is shown in Figure 4 and Figure 5 shows the simulation results of this execution.

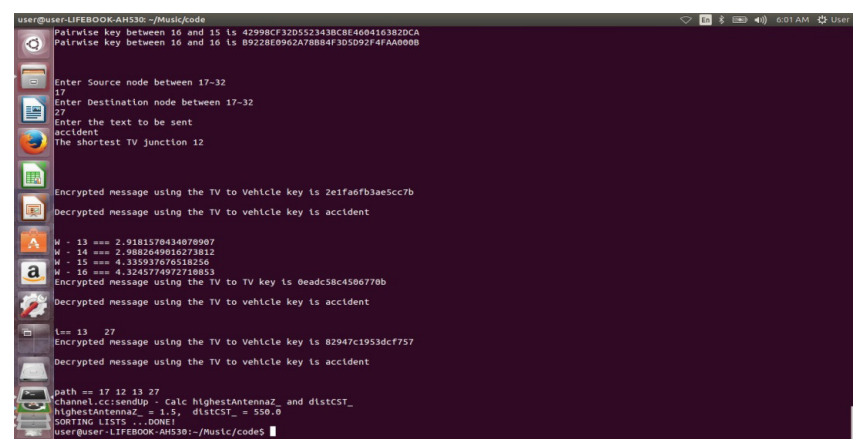

Figure 4. Execution phase.

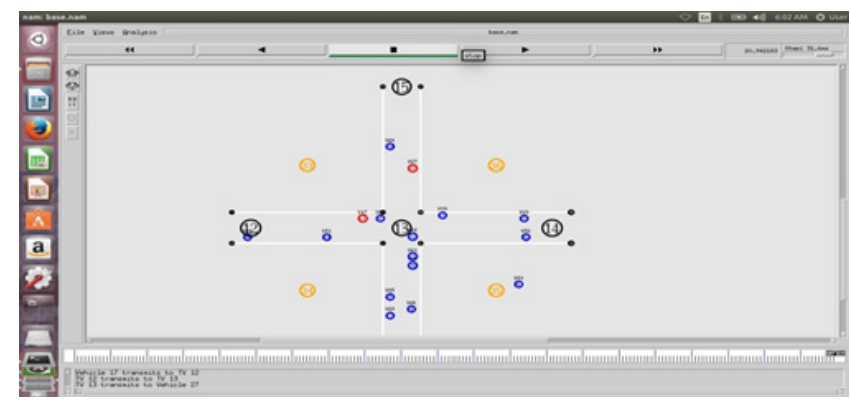

Figure 5. Execution result.

Here, the protocol has ensured that the data is being transmitted through authenticated vehicles and is traversed in shortest path to reach the destination at smallest time interval.

Using existing SIR protocol with QoS metrics, many possible paths from source to destination is obtained. From the possible paths, PSO algorithm is used to get the best optimal path, considering distance as the parameter. Use of PSO iteration in the proposed work is illustrated in Figure 6. 


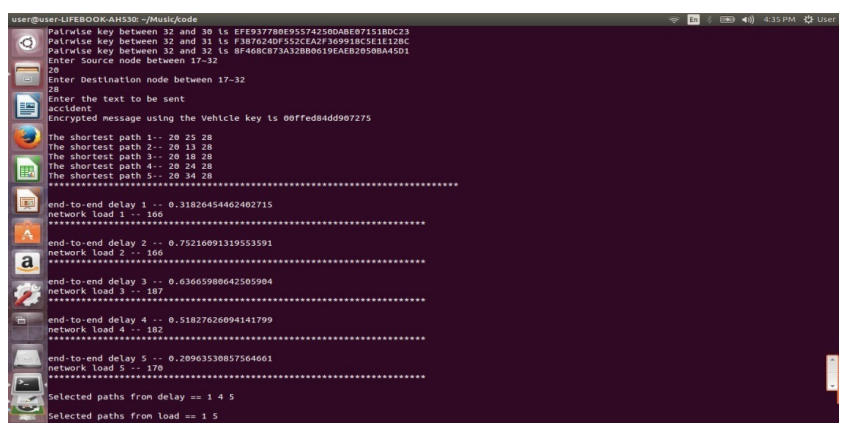

Figure 6. Using PSO iteration.

When the user enters the source and destination vehicle at the terminal, many possible paths from the source and destination is obtained. In the above example, the user has given 20 as the source vehicle and 28 as the destination vehicle. Five possible shortest paths from 20 to 28 are obtained. They are

Path 1 - 202528

Path 2 - 201328

Path 3 - 201828

Path 4 - 202428

Path 5 - 203428

From the obtained 5 paths, the algorithm will find the paths having delay less than the threshold value. Table 2 displays the delay values for the various paths.

Table 2. Delay values

\begin{tabular}{|c|c|}
\hline Path $1-202528$ & 0.318264 \\
\hline Path $2-201328$ & 0.752160 \\
\hline Path $3-201828$ & 0.636659 \\
\hline Path $4-202428$ & 0.518276 \\
\hline Path $5-203428$ & 0.209635 \\
\hline
\end{tabular}

From this, path-2 and path-3 are eliminated as it falls at upper bound. Thereby the paths considered for next phase are: path-1 -- 2025 28, path- 4 - 2024 28, path-5 203428 . From the selected 3 paths, the algorithm will find the paths having network load less than $70 \%$ of the entire network load. Table 3 shows the network load values calculated for the paths remaining after elimination from the previous phase.

Table 3. Network Load values

\begin{tabular}{|l|l|}
\hline Path $1-202528$ & 166 \\
\hline Path $4-202428$ & 182 \\
\hline Path $5-203428$ & 170 \\
\hline
\end{tabular}

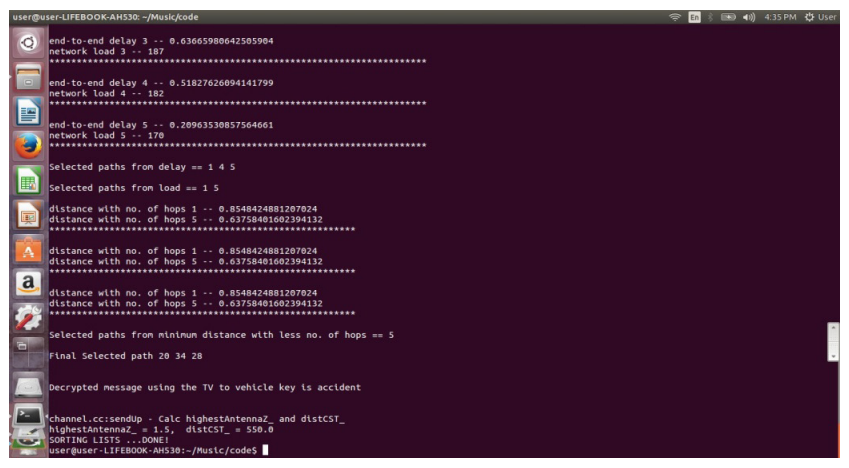

Figure 7. Using PSO iteration.

From this, path- 4 is eliminated as it falls at the upper bound.

Thereby we get 2 paths. They are: path-1 - 202528 and path-5 - 2034 38. Now Particle Swarm Optimization (PSO) is performed and this is illustrated in Figure 7.

Applying PSO to distance optimization over the above paths 1 and 5, the best possible path to reach the destination will be path-5 - 203428 . Since path-5 is having minimum distance with less number of hops when compared to path-1. Table 4 shows the distance values computed after applying PSO optimization.

Table 4. PSO iteration values

\begin{tabular}{|c|c|}
\hline Path $1-202528$ & 0.854842 \\
\hline Path $5-203428$ & 0.637584 \\
\hline
\end{tabular}

Hence from this we conclude that by using PSO, we get the best optimal path, where the data can be reached to the destination in shortest path.

\section{Result and Discussion}

The proposed work is evaluated with performance metrics - throughput, delay, drop, packet delivery ratio and residual energy levels.

Throughput increases marginally up to $1.7600 \mathrm{kbps}$ and then it steadily increases from $1.800 \mathrm{kbps}$. This shows that the throughput for the enhanced SIR (ESIR) with PSO algorithm is better than the existing SIR protocol and this comparison is shown in Figure 8.

There is a marginal increase of $10 \%$ from 69.000 to $70.000 \mathrm{kbps}$ and then it maintains the ratio till 3.000 seconds and then there is a gradual increase till 10.000 seconds for the enhanced protocol. This proves that the delivery rate is better for the enhanced SIR (ESIR) with PSO algorithm than the existing SIR protocol. The 


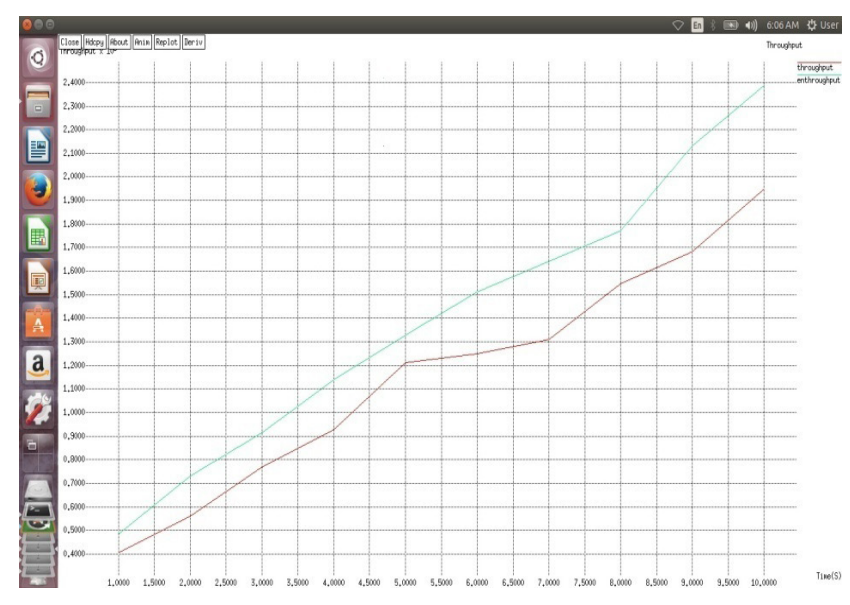

Figure 8. Throughput vs. Time.

increase in packet delivery ratio values with respect to time is shown in Figure 9.

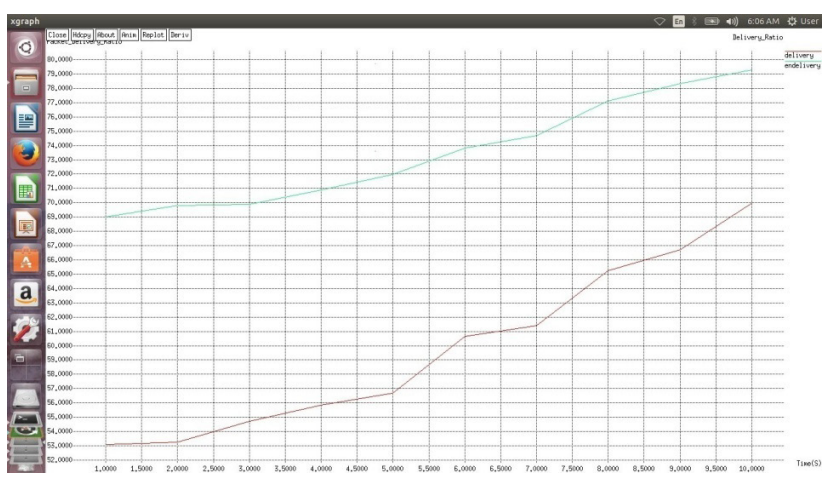

Figure 9. Packet delivery ratio vs. Time.

The energy utilized for enhanced SIR (ESIR) is gradually increasing and it converges at 4.000 milliseconds and then it increases marginally with the difference of $5 \%$ between the existing and proposed protocols. From Figure 10, we can determine that the energy utilization for enhanced SIR (ESIR) is less when compared with existing SIR protocol.

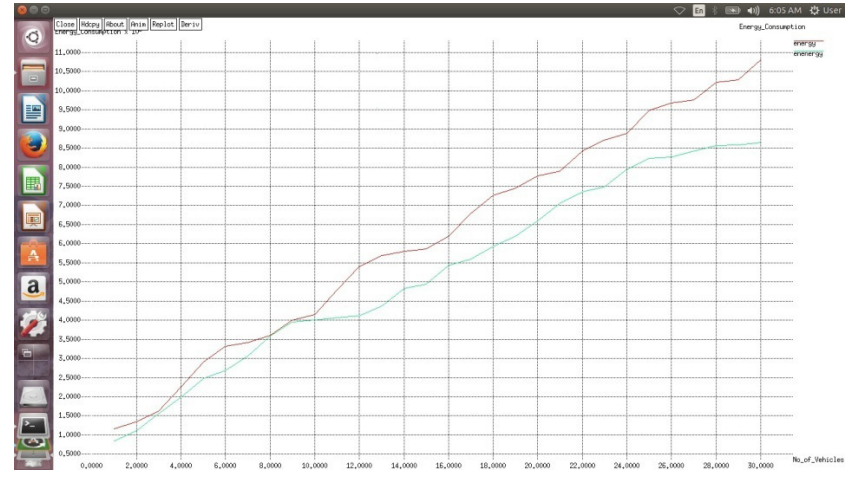

Figure 10. Residual energy levels vs. no. of vehicles.
Delay obtained for enhanced SIR with PSO is marginally increasing to a threshold of $1.000 \mathrm{~ms}$ and then steadily increases till $9.000 \mathrm{sec}$. This shows that delay for the enhanced SIR (ESIR) with PSO algorithm is better when compared to existing SIR protocol and this comparison is shown in Figure 11.

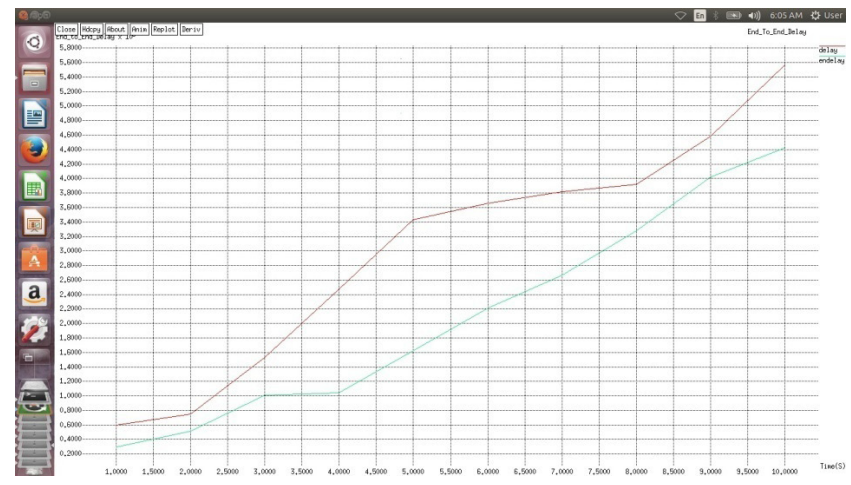

Figure 11. Delay vs. Time.

There is heavy packet drop in existing SIR protocol. This is due to the performance calculation of weight $\mathrm{W}$ by the TV. The packet drop for existing SIR protocol is starting from $56.000 \mathrm{kbps}$ and is gradually increasing till 10 milliseconds. But the Enhanced SIR (ESIR) starts from $45.000 \mathrm{kbps}$ and is gradually increasing. As shown in Figure 12 we can say that the existing SIR is having heavy packet drop than the enhanced SIR (ESIR) protocol.

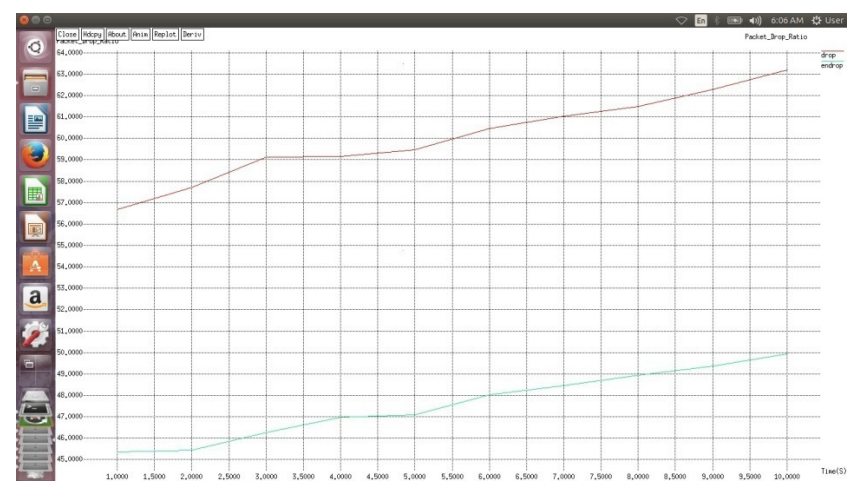

Figure 12. Packet Drop vs. Time.

\section{Conclusion}

SIR with PSO algorithm is the efficient method to transfer the data from source to destination in a secured and quickest path and the work could be enhanced by considering seamless scalability and Interoperability across heterogeneous platform. 


\section{References}

1. Bhoi SK, Khilar PM. SIR: a secure and intelligent routing protocol for vehicular ad hoc network. Institution of Engineering and Technology in Networks. 2015;4(3):185-94. https://doi.org/10.1049/iet-net.2014.0053

2. Li F, Wang Y. Routing in vehicular ad hoc networks: a survey. IEEE Vehicular Technologies. 2007; 2(2):12-22. https://doi.org/10.1109/MVT.2007.912927

3. Jerbi M, Senouci SM, Rasheed T, Ghamri-Doudane Y. Towards efficient geographic routing in urban vehicular networks. IEEE Transactions on Vehicular Technologies. 2009; 58(9):5048-59. https://doi.org/10.1109/TVT.2009. 2024341

4. Seet BC, Liu G, Lee BS, Foh CH, Wong KJ, Lee KK. A-STAR: a mobile ad hoc routing strategy for metropolis vehicular communications. Networking Technologies, Services, and Protocols, Performance of Computer and Communication Networks, Mobile and Wireless Communications. 2004; p. 989-99. https://doi.org/10.1007/978-3-540-24693-0_81

5. Securing the OLSR protocol. Available from: https://www. thomasclausen.net/wp-content/uploads/2015/12/securingolsr.pdf. Date accessed: 2003.
6. Perrig A, Canetti R, Tygar J, Song D. The TESLA broadcast authentication protocol. Crypto Bytes. 2002; 5(2):2-13.

7. Eiza MH, Owens T, Ni Q. Secure and Robust MultiConstrained QoS Aware Routing Algorithm for VANETs. IEEE Transactions on Dependable and Secure Computing. 2016; 13(1):32-45. https://doi.org/10.1109/TDSC.2014. 2382602

8. Wahab OA, Otrok H, Mourad A. VANET QoS-OLSR: QoSbased clustering protocol for vehicular ad hoc networks. Computational and Communication. 2013; 36(13):1422 35. https://doi.org/10.1016/j.comcom.2013.07.003

9. Adoption of Shortest Path Algorithm (Dijkstra's) in DistributedVANETUsingGRIDComputing. Availablefrom: https://www.researchgate.net/publication/261360489_ Adoption_of_Shortest_Path_Dijkastra_Algorithm_for_ Distributed_VANET_using_Grid_Computing. Date accessed: 12/2011.

10. Cryptography \& network security. Available from: https://onlinecourses.nptel.ac.in/noc19_cs28/preview. Date accessed: 2007.

11. Gayathri S, Radhika N. Greedy-Hop algorithm for detecting shortest path in Vehicular Networks. International Journal of Control Theory and Applications. 2016; 2(9):1125-33. 